\title{
Structure of the Insulin Receptor in Complex with Insulin using Single Particle CryoEM Analysis
}

Giovanna Scapin ${ }^{1^{*}}$, Venkata Dandey ${ }^{2 *}$, Zhening Zhang ${ }^{2}$, Winifred Prosise ${ }^{1}$, Alan Hruza $^{1}$, Corey Strickland ${ }^{1}$, Theresa Kelly, Todd Mayhood, Clinton S. Potter ${ }^{2}$ and Bridget Carragher ${ }^{2}$

1. Merck \& Co., Inc., Department of Biochemical Engineering \& Structure, 2000 Galloping Hill Rd. Kenilworth, NJ, 07033, USA

2. Simons Electron Microscopy Center, National Resource for Automated Molecular Microscopy, New York Structural Biology Center, 89 Convent Avenue, New York NY 10027

The insulin receptor (IR) is a homodimeric protein that plays a crucial role in controlling glucose homeostasis, regulating lipid, protein and carbohydrate metabolism, and modulating brain neurotransmitter levels. IR dysfunctions have been associated with a variety of diseases, including diabetes, cancer and Alzheimer's. The amino acid sequence of the IR monomer has been known since the 1980s; it is composed of an extracellular portion (ectodomain), a single transmembrane helix and an intracellular tyrosine kinase domain. Insulin binding to the dimer ectodomain triggers a signal leading to the kinase autophosphorylation and subsequent recruitment of downstream signaling molecules. Biochemical and mutagenesis data have identified two putative insulin binding sites (S1 and S2); S1 has also been characterized by structural studies. The monomeric ectodomain structure has been solved by $\mathrm{x}$-ray crystallography. However, the details of insulin binding to the full receptor and the mechanism of signal propagation are still not understood. We recently solved the single particle cryoEM reconstructions for the 1:2 (4.3 $\AA$ ) and 1:1 (7.4 $\AA$ ) IR ectodomain dimer:Insulin complexes, the first structures elucidating the binding mode of insulin to its receptor. In the presentation we will discuss the issues associated with sample preparation, data collection and processing, as well as the two structures and the suggested mechanism for downstream signal propagation. 